\title{
Vegeryday - Uma aplicação de auxílio à alimentação vegetariana
}

\author{
Giovana Santos ${ }^{1}$, Alex Mulattieri Suarez Orozco ${ }^{1}$, Alysson Hubner ${ }^{1}$, Ricardo Luis \\ dos Santos ${ }^{2}$ \\ ${ }^{1}$ Instituto Federal Sul-rio-grandense (IFSUL) - Sapucaia do Sul - RS - Brasil \\ ${ }^{2}$ Instituto Federal do Rio Grande do Sul (IFRS) - Vacaria - RS - Brasil \\ giovanadossantos97@gmail.com, \{orozco, alyssonhubner\}@inf.ufrgs.br, \\ ricardo.santos@vacaria.ifrs.edu
}

\begin{abstract}
The decision to start a vegetarian diet has become increasingly popular in Brazil. About $8 \%$ of the population in state capitals or metropolitan areas claim to be vegetarian. The reasons that lead people into vegetarianism are multidimensional, such as ethic, religion and dietary restrictions. However, the transition from omnivore to vegetarian becomes difficult for the individual. Sometimes, they don't not know which food to consume and can not maintain a balanced diet. This project seeks to help and assist these individuals to solve these problems through an easy access plataform that has a space where the user will register their diet, and doing so, be able to organize their diet and keep track of consumed nutrients.
\end{abstract}

Resumo. A decisão de começar uma alimentação vegetariana tem se tornado cada vez mais comum no Brasil. Cerca de $8 \%$ da população brasileira em capitais de estados ou regiões metropolitanas afirmam ser vegetarianos. Os motivos que levam ao vegetarianismo são multidimensionais, como ética, religião e restrições alimentares. No entanto, o processo de transição de onívoro para vegetariano se torna difícil para o indivíduo. Em alguns casos, o mesmo não sabe quais alimentos consumir. Desta forma, o presente projeto buscou auxiliar estes indivíduos a resolver estes problemas através de uma plataforma de fácil acesso, no qual o usuário pode cadastrar sua dieta semanal, sendo assim capaz organizar sua alimentação.

\section{Introdução}

A escolha de uma alimentação vegetariana em suas diversas formas tem sido adotada por diferentes grupos de pessoas. No Brasil, cerca de $8 \%$ da população em capitais de estados e regiões metropolitanas afirmam ser vegetarianos (IBOPE, 2012). Os motivos que levam o indivíduo ao vegetarianismo são multidimensionais, entre eles estão a ética e restrições alimentares. Partindo do momento desta escolha, o vegetariano possui dificuldades em manter uma dieta que supra todas as necessidades de aspecto nutricional que o organismo necessita, seja por falta de conhecimento de quais alimentos consumir, ou por falta de organização pessoal e controle da alimentação. Por isso, além de se situar no assunto, o adepto deve consultar profissionais da área da nutrição para definir uma base alimentar que forneça todos estes nutrientes. Com o crescimento da tecnologia em nossa sociedade, pode-se perceber um aumento de sites e 
portais com foco na difusão de informação a respeito do vegetarianismo. Porém, ao realizar pesquisas em redes sociais e através de conversas com vegetarianos, percebeuse que há uma carência de aplicações com o objetivo de auxiliar na transição e/ou manutenção diária da alimentação. Portanto, o presente projeto tem como objetivo auxiliar vegetarianos em sua dieta.

\section{Vegetarianismo e a Tecnologia}

No ano de 2016, o total de aplicativos nas lojas virtuais Google Play Store e Apple Store chegaram a dois milhões cada (STATISTA, 2016). O número alto de aplicações se deve ao constante questionamento de pessoas sobre como automatizar ou simplificar um processo diário. Em relação ao vegetarianismo não foi diferente, hoje é possível encontrar diversos aplicativos, porém, ainda não encontra-se uma aplicação que proporciona ao usuário controlar e visualizar a sua dieta dia a dia, que seja focado e exclusivo para vegetarianos.

A tecnologia pode, e deve ser usada para apoiar comunidades que precisam de maior visibilidade em nossa sociedade. Através de uma aplicação móvel, pode-se proporcionar ao usuário um diário onde ele irá controlar a sua alimentação e terá a visibilidade dos nutrientes que está consumindo, recebendo dicas sobre alimentos que deverá consumir para manter sua dieta equilibrada e saudável.

Com o objetivo de estudar as soluções presentes, foi realizada uma pesquisa sobre aplicações com temas e objetivos relacionados ao vegetarianismo. Foram selecionadas três soluções. São elas:

¿EsVegan? - uma plataforma em espanhol que através da leitura do código de barras do produto retorna a composição, informando se possui resquícios de animais (DESDELOSALVAJE, 2016).

HappyCow - uma plataforma que disponibiliza um guia de restaurantes vegetarianos ao redor do mundo, com informações de horários de funcionamento, contatos e endereços (TIME HAPPYCOW, 1999).

DietBox - uma plataforma que faz a intermediação entre paciente e nutricionista, disponibilizando um espaço para cadastramento de dietas (TIME DIETBOX, 2016).

Dentre as três soluções, nenhuma propõe as mesmas funcionalidades, de forma exclusiva para vegetarianos. Com isso, foi possível identificar um diferencial para o desenvolvimento de uma plataforma para este fim.

\section{Metodologia}

\subsection{Pesquisa de dados}

Para o desenvolvimento deste projeto foram realizadas pesquisas com o público alvo, através de formulários online, e pesquisas de artigos na área da nutrição. Através do formulário realizado com vegetarianos em redes sociais, foram obtidas 921 respostas, onde percebeu-se que a transição de onívoro para vegetariano torna-se difícil devido a falta de apoio de familiares e amigos (48,3\% dos entrevistados), assim como a falta de tempo para a organização e manutenção de uma dieta equilibrada (44,3\% dos entrevistados), como ilustrado na Figura 1. 


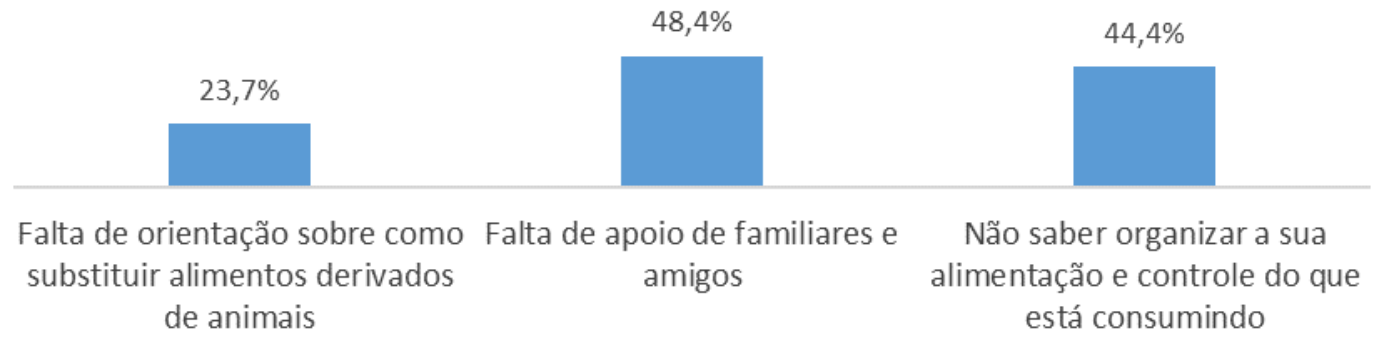

Figura 1. Gráfico das maiores dificuldades entre os vegetarianos

\subsection{Desenvolvimento e tecnologias}

Para suprir as necessidades do público consultado, foram definidas as seguintes funções do sistema: Auxiliar o usuário a organizar sua alimentação no dia a dia e ter o controle de nutrientes consumidos; Indicar alimentos de fácil preparação que possuem os nutrientes necessários para a manutenção da alimentação saudável; Dispor dados nutricionais dos alimentos apresentados, bem como as comparações entre os alimentos de origem animal a serem substituídos; Informar sobre profissionais da saúde próximos ao usuário, caso este queria um acompanhamento médico nutricional; Orientar o usuário na elaboração da sua dieta semanal, com informações nutricionais; Permitir que nutricionistas consigam manter um contato com seus pacientes, revisando suas dietas.

De forma a oferecer essas funcionalidades, foi desenvolvida uma plataforma denominada Vegeryday. Esta plataforma é composta por uma aplicação para o sistema operacional Android, com os dados persistidos no Sistema Gerenciador de Banco de Dados SQLite. Esta aplicação consome Web Services (LECHETA, 2015), desenvolvidos com o auxílio do framework Spring (PIVOTAL Software Inc, 2016). Para gerenciar a persistência dos dados dos Web services, foram utilizados o framework Hibernate (BAUER et alii, 2015) e o Sistemas Gerenciadores de Banco de Dados MySQL (ALLEN e OWENS, 2011). Algumas telas do aplicativo são demonstradas na Figura 2.

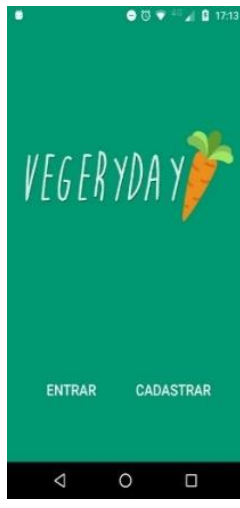

(A)

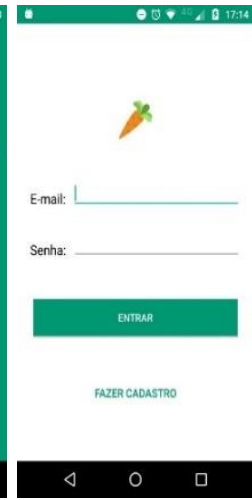

(B)

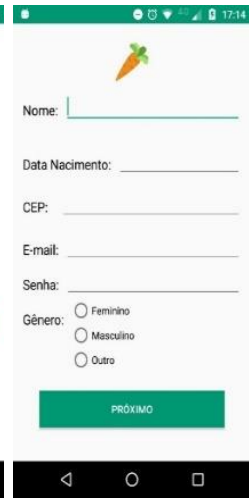

(C)

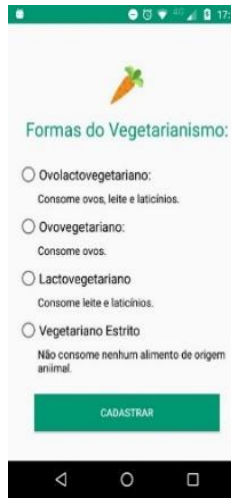

(D)

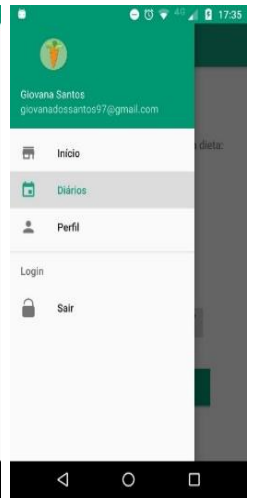

(E)

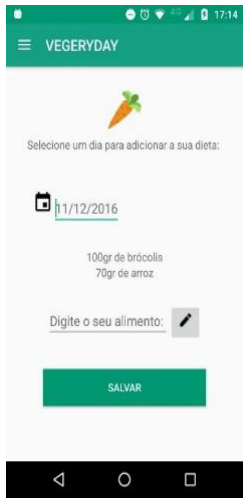

(F)

Figura 2. Telas do sistema: (A) Tela inicial, (B) Tela de Login, (C) Primeira tela de cadastro, (D) Segunda tela de cadastro com os tipos de vegetarianos,

(E) Menu do sistema, (F) Tela de cadastro de diários 


\subsection{Avaliação}

A plataforma foi avaliada através de formulário, o qual obteve-se 120 respostas, a fim de analisar a aceitação do projeto entre vegetarianos. Cerca de $97 \%$ dos entrevistados afirmaram que usariam a aplicação e acham necessário o desenvolvimento de aplicações voltadas para o vegetarianismo, como ilustrado na Figura 3.

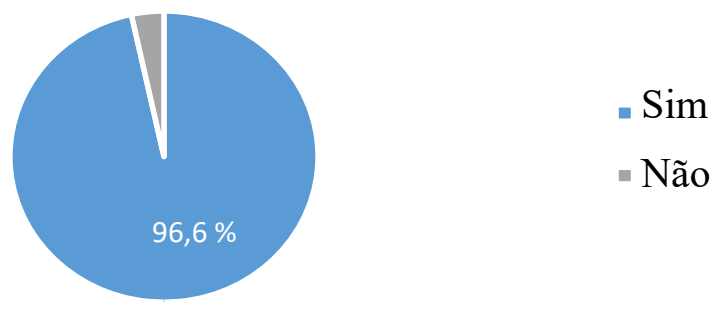

Figura 3. Gráfico de aceitação da aplicação junto ao público alvo

\section{Conclusão}

Este trabalho objetivou desenvolver uma plataforma para auxiliar pessoas a aderirem ao vegetarianismo. Através de uma pesquisa de dados, desenvolvimento da plataforma e pesquisa de aceitação, concluiu-se que o Vegeryday pode auxiliar seus usuários a manter suas dietas equilibradas, e também auxiliar uma comunidade a ter uma maior visibilidade dentro da sociedade

\section{Referências}

ALLEN, Grant e OWENS, Mike. (2011) "The Definitive Guide to SQLite". Apress, $368 p$.

BAUER, Christian; KING, Gavin; GREGORY, Gary. (2015) "Java Persistence with Hibernate". Em: Manning Publications Company, 608p.

DESDELOSALVAJE. (2016) “¿EsVegan?”. Disponível em: <http://esvegan.com/>. Acesso em: 19 mar. 2017.

DIETBOX. (2016) “DietBox”. Disponível em <https://dietbox.me/pt-BR/>. Acesso em: 19 mar. 2017.

HAPPYCOW. (1999) "HappyCow”. Disponível em: <https://www.happycow.net/about -us>. Acesso em: 19 mar. 2017.

IBOPE. (2012) "IBOPE 2012: 15,2 milhões de brasileiros são vegetarianos". Disponível em: <https://www.vista-se.com.br/ibope-2012-152-milhoes-de-brasileiros -sao-vegetarianos/>. Acesso em: 10 mar 2016.

LECHETA, Ricardo. (2015) "Web services RESTful: Aprenda a criar web services RESTful em Java na nuvem do Google". Novatec, 432p.

PIVOTAL Software Inc. (2016) “Spring for Android”. Packt Publishing, 126p.

STATISTA. Number of apps available in leading app stores as of June 2016. Disponível em: $\quad<$ https://www.statista.com/statistics/276623/number-of-apps-available-inleading-app-stores/>. Acesso em: 04 dez. 2016. 